\title{
Characteristics of Traveling-Wave Tubes with Periodic Circuits*
}

\author{
ROY. W. GOULD†
}

Summary-An analysis of an electron beam which interacts with a chain of coupled resonators is presented. Several impcrtant characteristics of traveling-wave tubes which employ periodic slowwave circuits are described. It is found that, even for a losisless circuit, the gain does not become large near either pass band edge although the interaction impedance does become very large. Furthermore, useful amplification is found to occur outside the normal circuit pass band, particularly when the frequency is below the lowfrequency cutoff where the circuit presents an inductive reactance to the beam.

The problem of matching uniform transmission lines to the periodic circuit is discussed from the equivalent circuit point of view and it is shown that the terminating impedance whick produces no reflection from the output end of the circuit wher the beam is present may be appreciably different from that recuired when the beam is absent.

The method of analysis applies to spatial harmonic operation, including backward spatial harmonics, as well as to synchrorously tuned multicavity klystrons.

\section{INTRODCCTION}

( ONSIDERABLE attention is being given to the design of traveling-wave tubes employing peniodic slow-wave circuits. Loaded waveguide circui's are of particular interest for high-power traveling wave amplifiers since they are capable of dissipating the considerable heat generated by intercepted electrons and offer the possibility of trading bandwidth for intersction impedance. ${ }^{1,2}$ The latter feature leads to a shorter tube for a given gain and a considerable saving in magnet weight. Several periodic slow-wave circuits are shown in Fig. 1. All of these circuits propagate slow electromanetic waves in certain bands of frequencies called the pass bands, and do not propagate electromagnetic wayes at other frequencies, the stopbands. The boundary between a pass band and stopband is called the cutoff frequency.

Much of this paper is focused on the behavior of traveling-wave tubes employing these types of slow.wave circuits, when the operating frequency is near one of the cutoff frequencies. To be specific, the slow-wave circuits of Fig. 1 are considered, although most of the results apply in a qualitative way to other periodic circuits. The widths of the lowest frequency pass band of these circuits are increased or decreased by increasing or decreasing the apertures between resonators. ${ }^{2}$

* Manuscript received by the PGED, November 11, 1967. This work was done while the author was at the Hughes Res, and Dev. Labs. Culver City, Calif.

$\dagger$ California Inst. Tech., Pasadena, Calif.

i M. Chodorow and F. J Nalos "The design of high-powe - traveling-wave tubes," Proc. IRE, vol. 44, pp. 649-659; May, 1966.

$2 \mathrm{M}$. Chodorow and R. A. Craig, "Some new circuits for highpower traveling-wave tubes," PRoc. IRE, vol. 45, pp. 1106-1118; August, 1957.

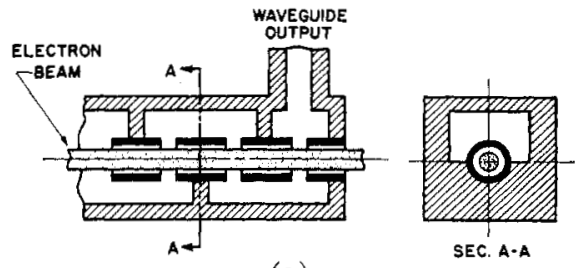

(a)

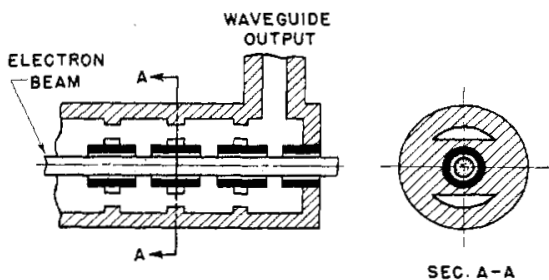

(b)

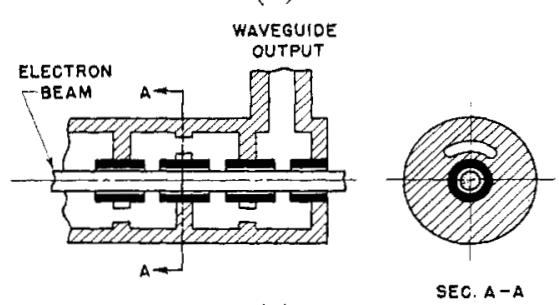

(c)

Fig. 1-Several periodic slow-wave circuits for traveling-wave tubes.

The Pierce theory of traveling-wave interaction ${ }^{3}$ assumes a slow-wave circuit which propagates two waves, one in the $+z$ direction and one in the $-z$ direction. If this theory is applied to an electron beam inter acting with one of the spatial harmonics, or fundamental, of a periodic slow-wave circuit, the interaction impedance

$$
K=\frac{E^{2}}{2 \beta^{2} \bar{P}}
$$

becomes very large when the frequency approaches a cutoff frequency of the circuit. This is because the power flow $P$ is given by

$$
P=W v_{g},
$$

where $W$ is the energy stored per unit length, and $E^{2} / W$ changes slowly with frequency in the vicinity of a cutoff but the group velocity of the wave, $v_{a}$, approaches zero.

Since the interaction parameter $C$ approaches infinity, and the gain per unit length is proportional to $C$, it appears that the small-signal gain should become very large at each cutoff frequency providing approximate

${ }^{3}$ J. R. Pierce, "Traveling Wave Tubes," D. Van Nostrand Co., Inc., New York, N. Y.; 1950 . 
synchronism between the electrons and the wave prevails. In addition, it should be remembered that the reflection coefficient of the junction between such a periodic circuit and a smooth waveguide or a coaxial line must necessarily approach unity as a cutoff frequency is approached. Thus at the same time that the gain increases, the input and output transitions become reflecting and unless a large amount of attenuation is provided for the wave reflected at the output transition, oscillation seems inevitable. The inclusion of circuit loss, which increases sharply near the cutoff frequencies, does not grossly alter this picture. But, the presence of two spatial harmonics with nearly the same phase velocity does have a pronounced effect. It is shown here that when this interaction with two spatial harmonics is included, finite gain at the cutoff frequencies generally results and useful amplification can sometimes be obtained in the stopband. The starting current for backward-wave oscillations on adjacent backward spatial harmonics no longer approaches zero as the frequency approaches either of the cutoff frequencies.

There are at least two ways in which one might proceed to analyze this situation. The first is to modify Pierce's normal mode theory ${ }^{4}$ to account for interaction with two nearly synchronous propagating modes-one forward and one backward. In the second, the one used here, the distributed periodic slow-wave circuit is replaced by an equivalent lumped periodic circuit. This seems to be a good approximation for the slow-wave circuits shown in Fig 1(a) and 1(b). The electron beam sees a series of relatively short gaps separated by nearly field-free drift regions. Certain aspects of this analysis are similar to the analysis by Pierce and $\mathrm{Wax}^{5}$ of filter-type traveling-wave tubes except that space charge and boundary conditions are included here. The analysis also is carried through numerically for a variety of cases. For the numerical work, it is necessary to consider a very specific lumped equivalent circuit.

The system of Fig. 1 looks very much like a multicavity klystron in which each resonator is coupled to adjacent resonators. This similarity leads to the schematic representation of the traveling-wave tube shown in Fig. 2. Several equivalent representations of the slow-wave circuit are obtainable from this one by using the equivalent representations of the mutual inductance. Although it is not possible to represent a distributed circuit exactly by lumped frequency independent elements as shown, this representation contains all the essentials of the problem and it is thought to be an adequate approximation to the actual circuit. The coupling between adjacent resonators is taken to be inductive because the coupling holes are located in a region where the magnetic field is strong and the electric field is relatively weak. No attempt has been made to calculate the values of $L, G, C$, and $M$ theo-

\section{${ }^{4}$ Ibid., ch. 7 .}

6. R. Pierce and N. Wax, "A note on filter type traveling wave amplifiers," Proc. IRE, vol. 37 , pp. 622-625; June, 1949.

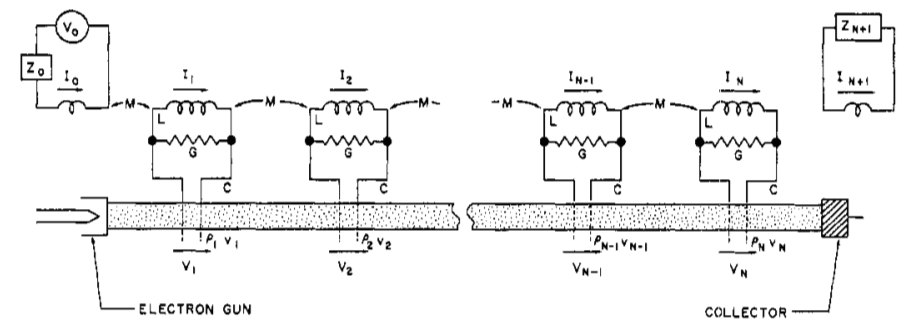

Fig. 2-Equivalent circuit of periodic traveling-wave tube.

retically from the actual dimensions of the circuit, but instead they are chosen in the following manner.

1) The midband frequency $\omega_{0}=1 / \sqrt{L / C}$ is chosen to agree with the measured center frequency of the actual slow-wave circuit.

2) The relative width of the pass band determined by the coupling coefficient, $k=M / L$, is chosen to give agreement with the measured bandwidth of the actual slow-wave circuit.

3) The interaction impedance determined by $\sqrt{\bar{L}} / \bar{C}$ and $k$ is chosen to give agreement with the measured impedance of the slow-wave circuit at midband.

4) The conductance $G$ is chosen so as to make the midband attenuation agree with the measured attenuation. In most cases the effect of loss is negligible and $G$ may be set equal to zero.

The equivalent circuit used in this analysis is also a suitable representation for the slow-wave circuit described by Chodorow and Nalos, Fig. 1(c), since the coupling between cavities is also primarily inductive. The equivalent circuit proposed here differs slightly from the one suggested by Chodorow and Nalos ${ }^{1}$ but these differences would be important only for wide-band circuits, where the use of frequency-independent elements in the equivalent circuit may be questionable.

\section{The System of Equations}

The following equations describe the lumped circuit elements of the $n$th resonator in Fig. 2.

$$
\begin{gathered}
V_{n}=j \omega L I_{n}+j \omega M\left(I_{n-1}+I_{n+1}\right) \\
I_{n}+(G+j \omega C) V_{n}=i_{n}
\end{gathered}
$$

where current in the electron beam at the $n$th gap $i_{n}$ also flows through the external circuit. Throughout this analysis, it is assumed that the gap transit angle is small. It is possible, of course, to include gap transit time effects approximately by using the Llewellyn-Peterson equation to describe the gap region. The principal modification would be to introduce the square of the gap factor into expression for the coupling constant $\kappa$ which is defined later. This is probably an unnecessary refinement in the analysis.

The modulation of the electron beam at the exit of the $n$th gap can be related to the modulation of the beam at the exit of the preceding gap 


$$
\begin{aligned}
i_{n} & =A_{11} i_{n-1}+A_{12} \rho_{0} v_{n-1} \\
\rho_{0} v_{n} & =A_{21} i_{n-1}+A_{22} \rho_{0} v_{n-1}+\frac{\rho_{0}}{u_{0}} \frac{e}{m} V_{n}
\end{aligned}
$$

where

$$
\begin{aligned}
& A_{11}=e^{-j \theta_{e}} \cos \theta_{\alpha} \\
& A_{12}=j e^{-j \theta_{\sigma}} \frac{\omega}{\omega_{q}} \sin \theta_{q} \\
& A_{21}=j e^{-j \theta_{e}} \frac{\omega_{q}}{\omega} \sin \theta_{q} \\
& A_{22}=e^{-j \theta_{e}} \cos \theta_{q}
\end{aligned}
$$

and

$$
\theta_{e}=\frac{\omega l}{u_{0}} \quad \theta_{a}=\frac{\omega_{q} l}{u_{0}} .
$$

$\rho_{0}$ is the $\mathrm{dc}$ or average charge density per unit length in the electron beam; $\omega_{q}$ is the reduced electron plasma frequency, and $u_{0}$ is the electron drift velocity. The elements of the $A$ matrix have been obtained thro tgh a straightforward application of space-charge wave theory. It is also assumed that the alternating component of beam current is unchanged in passing through th gap and that the alternating component of velocity is increased by an amount proportional to the gap voltage. This result follows directly from the Llewellyn-Peterson equations for a thin gap and negligible space charge, or from simple physical considerations.

The above circuit equations apply only to resonators. Separate equations must be written for the input circuit and the output circuit.

$$
\begin{aligned}
Z_{0} I_{0}+j \omega M I_{1} & =V_{0} \\
Z_{N+1} I_{N+1}+j \omega M I_{n} & =0 .
\end{aligned}
$$

The impedances $Z_{0}$ and $Z_{N+1}$ are assumed to contain the effect of the transitions as well as the internal imperance of the generator and the load impedance.

The current and velocity modulation of the electron beam upon entering the first gap is zero since it has not been acted upon by the microwave field. The following two equations describe these two boundary conditions.

$$
\begin{array}{rlrlrl}
i_{0} & =0 & \text { or } & i_{1} & =0 \\
\rho_{0} v_{0}=0 & \text { or } & \rho_{0} v_{1}-\frac{\rho_{0} e}{u_{0} m} V_{1} & =0 .
\end{array}
$$

Eqs. (3) through (15) completely describe the system seen in Fig. 2.

\section{Properties of the Slow-Wave Circutt in th:} Absexce of the Electron Beam

It is instructive to consider the circuit characteristics separately before solving the complete problem. Only (3) and (4) (with $i_{n}=0$ ) are required to find the characteristic waves of the circuit. Eqs. (3) and (4) actually form a set of difference equations. This set of difference equations has certain characteristic solutions in which both the current and voltage in a particular resonator differ from the current and voltage in the previous resonator by a multiplicative constant, $\mu$. Thus

$$
I_{n}=\mu^{n} I_{0} \quad V_{n}=\mu^{n} V_{0}
$$

The attenuation per resonator $\alpha$ measured in nepers and the phase shift per resonator $\theta$, measured in radians, is related to the constant $\mu$ in the following way:

$$
\mu=e^{-(\alpha+j \theta)} .
$$

In order for the equations to have solutions of the form (16), the constant $\mu$ must satisfy

$$
\lambda(\lambda-j q)(1+k \mu+k / \mu)=1
$$

where the following dimensionless or normalized parameters are employed.

$$
\omega \sqrt{L C}=\lambda,
$$

the normalized frequency

$$
\frac{M}{L}=k,
$$

the resonator coupling coefficient

$$
q=G \sqrt{L / C}
$$

the reciprocal of the resonator $Q$.

For positive $k$ the fundamental branch of the Brillouin diagram is a forward spatial harmonic, while for negative $l$ the fundamental branch of the Brillouin diagram is a backward spatial harmonic. ${ }^{\theta}$ The circuit phase and attenuation characteristics are illustrated in Fig. 3. When the solutions of (18) are expressed in terms of $\alpha$ and $\theta$ as defined by (17), $\theta$ is determined only to within an artibrary multiple of $2 \pi$. The solutions. $\theta-2 \pi, \dot{\theta}, \theta+2 \pi$, $\theta+4 \pi, \cdots$ represent exactly the same physical situation since $\mu$ is the same for each, as are the currents and voltages. However, it is interesting to note that formally, at least, these additional solutions can be interpreted as the phase shifts per section of the spatial harmonics in expansions of the fields along the axis of the tube. The upper and lower cutoff frequencies are given by

$$
\begin{aligned}
& \lambda_{2}=\frac{1}{\sqrt{1-2|k|}} \\
& \lambda_{1}=\frac{1}{\sqrt{1+2|k|}} .
\end{aligned}
$$

The fractional bandwidth is approximately $2|k|$ as long as $|k|$ is small. When $k$, and hence $M$, is negative, the phase characteristics given by (18) agree very well with those obtained with the circuit of Fig. 1(a) and 1(b). For frequencies greater than that given by (22) there are two

${ }^{6}$ It follows from simple physical considerations that $M$ is negative for the circuit of Fig. 1(a). The sign convention for the mutual inductance used here is opposite from that used in references 1 and 2 . 

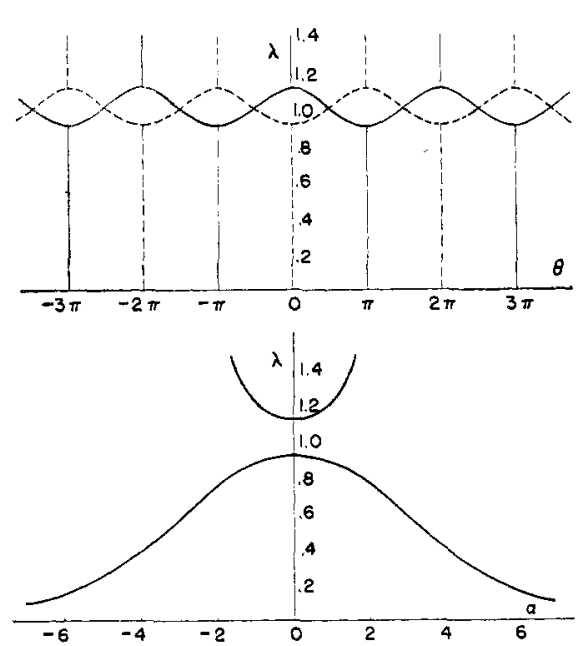

Fig. 3-Attenuation and phase characteristics of the slow-wave circuit. $k=-0.1$, solid line; $k=+0.1$, dashed line.

evanescent waves with phase shifts of zero radians per resonator. For frequencies below that given by (23) there are two evanescent waves whose phase shift is $\pi$ radians per resonator. These solutions are indicated by the vertical lines in Fig. 3. The pass band is centered approximately about $\lambda=1$.

When the resonators are lossless, $q=0$, there is no attenuation in the pass band and $\alpha=0$. When the resonators are lossy, the attenuation per resonator is given approximately by

$$
\begin{aligned}
\alpha & =\frac{q}{2|k|} \text { midband, } \lambda=1 \\
& =\left(\frac{q}{2|k|}\right)^{1 / 2}(1+2|k|)^{3 / 4} \text { upper cutoff, } \lambda=\lambda_{2}{ }^{7} \\
& =\left(\frac{q}{2|k|}\right)^{1 / 2}(1-2|k|)^{3 / 4} \text { lower cutoff, } \lambda=\lambda_{1}{ }^{7}
\end{aligned}
$$

The variation with frequency is shown in Fig. 4.

The interaction impedance $K$ of the slow-wave circuit may be evaluated as follows. When the electric field along the electron beam is resolved into a series of spatial harmonics, each harmonic has the same amplitude, $V_{n} / l$, where $V_{n}$ is the voltage across the $n$th gap and $l$ is the distance between gaps. This result holds as long as the gap spacing $h$ is small compared with the distance between gaps. When the gap spacing is not small, the spatial harmonic amplitude is reduced by the gap factor

$$
\frac{\sin \left(\theta \frac{h}{2 l}\right)}{\left(\frac{\theta h}{2 l}\right)} \text {. }
$$

The average power $P$ transmitted along the circuit is given by

$$
P=\frac{1}{2} \operatorname{Re}\left(j \omega M I_{n-1} I_{n}^{*}\right)
$$

${ }^{7}$ Chodorow and Craig's (16), which implies that the attenuation becomes infinite at a cutoff, does not apply near a cutoff frequency.

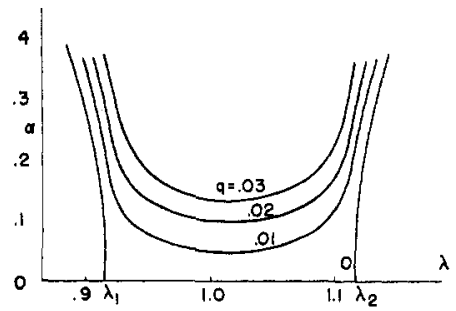

Fig. 4-Variation of attenuation, constant with frequency when $|k|=0.1, q=0,0.01,0.02,0.03$.

Using (4) with $G=0$ and $i_{n}=0$, this may be written

$$
P=\frac{1}{2} \operatorname{Re}\left(j \omega^{3} M C^{2} V_{n-1} V_{n}^{*}\right) \text {. }
$$

Since

$$
V_{n-1}=V_{n} e^{i \theta}
$$

this result may be written

$$
\frac{V_{n}^{2}}{2 P}=-\frac{\sqrt{L / C}}{\lambda^{3} k \sin \theta} .
$$

The interaction impedance $K$ then is given by

$$
K=\frac{E^{2}}{2 \beta^{2} P}=\frac{\sqrt{L / C}}{\lambda^{3} k \theta^{2} \sin \theta} .
$$

The dependence of the interaction impedance on the spatial harmonic number is contained within the function $\theta^{2} \sin \theta$. Note that $K$ is infinite at $\theta=0, \pi, 2 \pi, \cdots$, i.e., at the pass band edges. The midband interaction impedance is inversely proportional to bandwidth because of the factor $k$ appearing in the denominator.

The input impedance of this circuit is easily shown to be

$$
\begin{aligned}
& Z_{i n}=Z_{0}+j \omega M e^{-(\alpha+i \theta)} \\
& R_{i n}=R_{0}+\omega M e^{-\alpha} \sin \theta \\
& X_{i n}=X_{0}+\omega M e^{-\alpha} \cos \theta
\end{aligned}
$$

where $\theta$ is the phase shift per section of a wave traveling to the right. When $G$ is zero and $M$ is positive, $\theta$ varies between 0 and $\pi$ as the frequency is increased from the low-frequency cutoff to the high-frequency cutoff and $\alpha$ is zero. The input resistance starts at 0 , reaches a maximum near midband, and then decreases to 0 , while the reactance is first positive, goes through zero near midband, then becomes negative. When $M$ is negative, $\theta$ varies between $\pi$ and $2 \pi$ as the frequency is increased from the lowfrequency cutoff to the high-frequency cutoff, causing the input resistance and reactance variations to be the same as for positive $M$. For either positive or negative $M$ when the frequency is below the lower cutoff frequency, the input resistance is zero and the input reactance is positive. When the frequency is above the upper cutoff frequency the input resistance is again zero and the reactance is negative. Fig. 5 is a sketch of the normalized resistance and reactance as a function of frequency for $k=-.1, q=0, R_{0}=0$, and $X_{0}=0$. It is evident from Fig. 5 that there is considerable input reactance which, if eliminated, would result in a much better transition to 

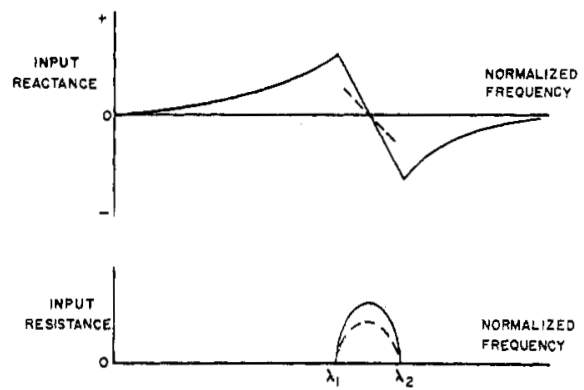

Fig. 5-Input resistance and reactance of the slow-wave circuit, without electron beam (solid curves) and with electrcn beam (dashed curves).

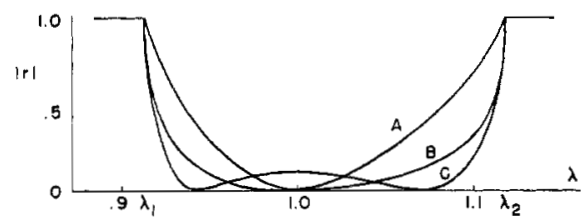

Fig. 6-Reflection coefficient at input or output of the slow-wave circuit as a function of normalized frequency, $A$, no reactance compensation. $B$, reactance compensation with resistance natched at midband. $C$, reactance compensation with resistance nlatched at two frequencies.

the transmission line of nearly constant characteristic impedance. At the terminals of a slow-wave cireut and at multiples of half-guide wavelength along the input transmission line the reactance changes from ind uctive to capacitive as frequency is increased. This reactance change can be compensated by placing a series resonant circuit in series with the transmission line at any of these points. Fig. 6 shows the reflection coefficient whic 1 can be obtained if the reactance is completely eliminated in the pass band. The remaining reflection arises from the mismatch of the resistance of the circuit to that of a transmission line. Because of the shape of the resistance curve, it generally is possible to match the transmission line at two frequencies. Fig. 6 also shows the reflection coefficient when this method is employed.

The equivalent circuit analysis also suggests other methods of eliminating the reactance variation in the pass band, some of which are seen in Fig. 7. Fig. 7(a) shows the method just described, that of the external resonator. A half-cavity may be used between the uniform transmission line and the periodic cireuit as illustrated by Fig. 7(b). Still another method is shown in Fig. 7(c) where the coupling between the last and next-to-the-last resonator is reduced by a factor of 2 .

\section{Properties of the Slow-Wave Circetit and the Electron Beam}

In this section, the characteristic waves of the combined slow-wave circuit and the electron beam are studied. The nature of the characteristic waves is discussed and the relative excitation of the various waves is determinet by the boundary conditions of the problem. To solve the equations, it is convenient to introduce several more dimensionless parameters. (a)

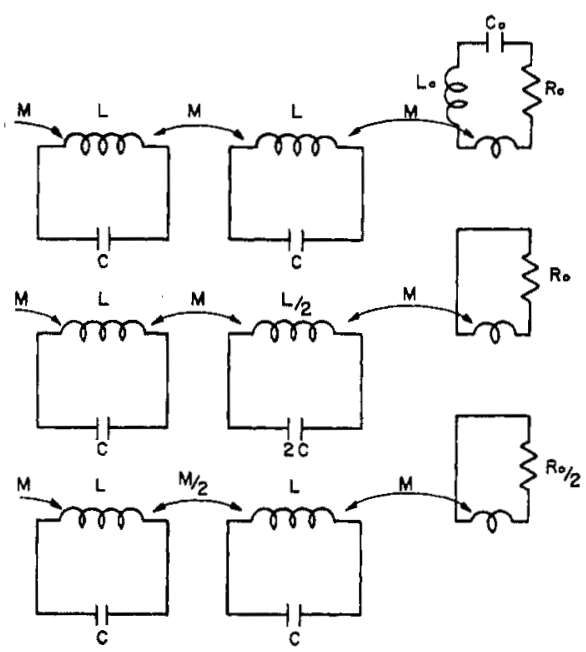

Fig. 7-Three methods of minimizing reflection at junction between the slow-wave circuit and a uniform transmission line. (a) External resonator. (b) Half section. (c) Modified coupling.

$$
V_{n}^{0}=\sqrt{C / L} V_{n}, \begin{aligned}
& \text { normalized voltage across } \\
& n \text {th resonator }
\end{aligned}
$$

a dimensionless constant

$\kappa=\frac{e\left\lfloor p_{0} \mid\right.}{m u_{0}} \sqrt{L / C}$, which measures the coup-

beam and slow-wave circuit

$$
Z_{0}^{0} \quad \frac{Z_{0}}{\sqrt{L / C h}}, \quad \text { the normalized impedance }
$$

$$
Z_{N+1}^{0}=\frac{Z_{N+1}}{\sqrt{\bar{L} / \bar{C}}}, \quad \text { the normalized impedance }
$$$$
Z_{N+3}^{0}=\frac{Z_{N-1}}{\sqrt{L / C k}}, \quad \text { of the output circuit }
$$

$$
V_{0}^{0}=\frac{V_{0}}{\sqrt{L / C k}}, \quad \text { the normalized input volt- }
$$

$$
\theta_{0}=\omega_{0} l / u_{0}, \quad \begin{aligned}
& \text { midband transit angle } \\
& \text { between resonators. }
\end{aligned}
$$

When written in terms of the normalized variables, (3) to (6) become

$$
\begin{aligned}
V_{n}^{0} & =j \lambda\left(I_{n}+k I_{n-1}+k I_{n+1}\right) \\
i_{n} & =I_{n}+(j \lambda+q) V_{n}^{0} \\
i_{n} & =A_{11} i_{n-1}+A_{12} i_{n-1}^{\prime} \\
i_{n}^{\prime} & =A_{21} i_{n-1}+A_{22} i_{n-1}^{\prime}-k V_{n}^{0}
\end{aligned}
$$

where

$$
\begin{array}{ll}
A_{11}=e^{-i \lambda \theta_{0}} \cos \theta_{a} & A_{21}=j e^{-i \lambda \theta_{0}} \frac{\theta_{a}}{\lambda \theta_{0}} \sin \theta_{q} \\
A_{12}=j e^{-j \lambda \theta_{0}} \lambda \frac{\theta_{0}}{\theta_{a}} \sin \theta_{\alpha} & A_{22}=e^{-i \lambda \theta_{0}} \cos \theta_{a}
\end{array}
$$

and $i_{n}^{\prime}$ is written for the quantity $\rho_{0} v_{n}$. Similarly, the equations for the input and output circuits become

$$
\begin{aligned}
V_{0}^{0} & =j \lambda I_{1}+Z_{0}^{0} I_{0} \\
0 & =j \lambda I_{N}+Z_{N+1}^{0} I_{N+1} .
\end{aligned}
$$


To find the characteristic waves of the system, assume that each ac quantity varies from section to section in the manner suggested by (16). Eqs. (40) and (41) may be combined then to give the circuit equation

$$
\frac{i_{n}}{V_{n}^{0}}=\frac{1-\lambda(\lambda-j q)(1+k \mu+k / \mu)}{j \lambda(1+k \mu+k / \mu)}
$$

while (42) and (43) may be combined to give the electronic equation

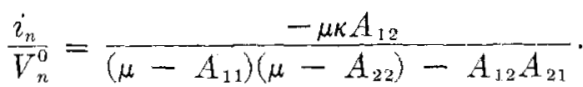

In order to have solutions of the assumed form, the righthand side of (46) must be equal to the right-hand side of (47). The equation obtained in this way is easily rewritten as a polynomial of fourth degree in $\mu$. Thus, there are four characteristic waves. Under some conditions these may be identified as two circuit waves and two space-charge waves. Denoting the four solutions of the fourth-degree polynomial in $\mu$ by $\mu_{1}, \mu_{2}, \mu_{3}$, and $\mu_{4}$, the current in the $n$th cavity may be written

$$
I_{n}=\sum_{r=1}^{4} I_{0 r} \mu_{r}^{n}
$$

$I_{01}, I_{02}, I_{03}$, and $I_{04}$ are constants which measure the amplitude of each wave, and they are determined by requiring the solution to satisfy the boundary conditions of the problem expressed by (14), (15), (44), and (45). The normalized resonator voltage and the current in the electron beam are given by

$$
\begin{aligned}
V_{n}^{0} & =j \lambda \sum_{r=1}^{4}\left(1+k \mu_{r}+k / \mu_{r}\right) I_{0 r} \mu_{r}^{n} \\
i_{n} & =\sum_{r=1}^{4}\left[1+j \lambda(j \lambda+q)\left(1+k \mu_{r}+k / \mu_{r}\right)\right] I_{0 r} \mu_{r}^{n} .
\end{aligned}
$$

\section{Results}

Fig. 8 shows one of the first-gain curves obtained using this method of calculation. The external resonator matching scheme of Fig. 7 (a) was used in both the input and output circuits, and yet large gain variations were obtained as shown by the solid line. These variations in gain are due to residual reflections at the input and output circuits, although the latter were chosen to minimize the reflection with the electron beam absent. The input and output impedances of the slow-wave circuit are altered sufficiently by the presence of the electron beam to explain this result. The output impedance ${ }^{8}$ is given by

$$
Z_{\mathrm{out}}=j \omega M e^{-\left(\alpha_{1}+j \theta_{1}\right)}
$$

where $\alpha_{1}$ and $\theta_{1}$ are the attenaution constant and phase constant of the growing wave. In a typical case where the gain per resonator is $3 \mathrm{db}, \alpha_{1}=-0.35$ and the output

${ }^{8}$ This is the impedance which matches the growing wave; i.e., when the growing wave is incident upon the output transition, this impedance completely absorbs it, producing no reflection.

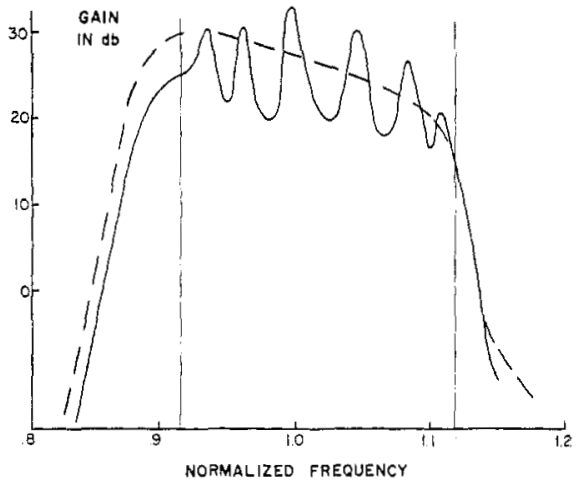

Fig. 8-Gain vs frequency of a nine-resonator traveling-wave tube. $\kappa=0.0118, k=-0.1, q=0, \theta_{0}=1.32 \pi, \theta_{0}=0.18 \pi$. Solid curve: terminating impedances chosen to minimize reflections without beam. Dashed curve: terminating impedances chosen to minimize reflections with beam.

impedance may be reduced by a factor of 1.4 . The variation of the resulting output impedance with frequency is indicated by the dashed line in Fig. 5. In subsequent gain calculations the constants $L_{0}, C_{0}$, and $R_{0}$ of Fig. 7 (a) were chosen so as to best approximate the impedance given by (51). The input circuit impedance was made the same as the output circuit impedance. The dashed curve of Fig. 8 shows the gain calculated with this assumption as to input and output circuit impedances. The absence of rapid variations in gain signifies that the input and output circuits now match the slow-wave circuit and electron beam very well. Another very important result appears in Fig. 8. Amplification exists outside the normal pass band of the slow-wave circuit. The effect is much more significant near the low-frequency cutoff where the impedance presented by the slow-wave circuit to the electron beam is inductive.

Solutions of the type in Fig. 8 are obtained in the following way. The constants $\kappa, k, q, \theta_{0}$, and $\theta_{p}$ are determined by the slow-wave circuit and electron beam under consideration. The constant $\kappa$ is related to the interaction impedance and the beam current and beam voltage, through (32) and (35) and is chosen to give the correct midband interaction parameter $C$. The coefficient of coupling $k$ is chosen to give the correct fractional bandwidth and the loss parameter $q$ is chosen to give the correct midband attenuation. $\theta_{0}$ is the midband transit angle through one period of the slow-wave circuit and is determined by the circuit dimensions and the operating voltage. For approximate synchronism with the strongest forward spatial harmonic of the slow-wave circuit of Fig. 1 (a), $\theta_{0}$ should be approximately $3 \pi / 2 . \theta_{\alpha}$ is related to the reduced plasma frequency, the period of the slow-wave circuit and the electron velocity, as defined in (11). Once these constants are specified, the fourth-degree polynomial in $\mu$ may be solved for various values of $\lambda$, that is, as a function of the operating frequency. Results of this type of calculation are presented in Fig. 9.

The upper half of Fig. 9 shows the phase shift per section as a function of the normalized frequency. The two coordinates are interchanged in order that the 

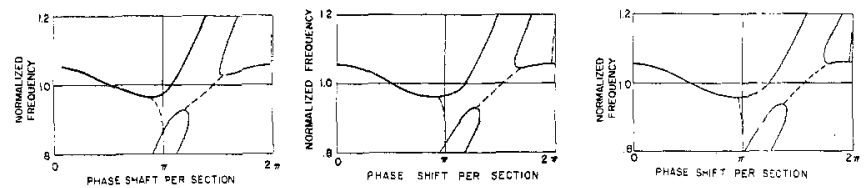

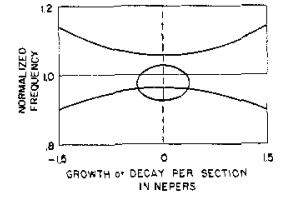

(a)

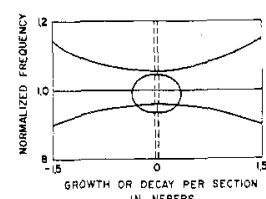

(b)

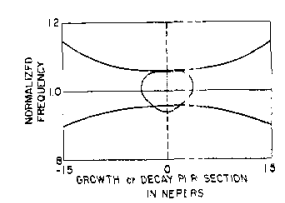

(c)
Fig. 9-Phase and attenuation characteristics of the traveling-wave tube. $k=-0.05, q=0, \kappa=0.0033, \theta_{q}=0.13 \pi, \theta_{0}:=1.28 \pi$ (left), $1.36 r$ (center), $1.44 \pi$ (right). Solid lines indicate a single root, and dashed lines indicate double root of the charecteristic equation.

diagram resemble the usual Brillouin diagram for the slow-wave circuit. Because of the presence of the electron beam, there are now four waves at each frequency. The diagram is periodic in $\theta$ with period $2 \pi$ and for convenience only those solutions for which $\theta$ lies between 0 and $2 \pi$ are shown. A solid line depicts a constant am olitude wave $(\alpha=0)$ while a dashed line depicts a growi g and decaying pair of solutions in which $\alpha$ has equil and opposite values. The attenuation constant $\alpha$ for the growing and decaying wave solutions is plotted in the lower half of the figure. At frequencies well belciw the lower cutoff frequency, $(\lambda \leq 0.8)$ and at frequenci:s well above the upper cutoff frequencies, $(\lambda \geq 1.2)$, the four solutions represent two evanescent circuit waves and a slow and a fast space charge wave. Within the pass band, circuit waves may be coupled to the space-charge waves in a manner which makes it impossible to identify the solutions as clearly of one type or another. For example, the slow space-charge wave couples to the forward 1;raveling circuit wave to give rise to a growing and deraying pair of waves; the former is responsible for amplification. This pair of solutions corresponds to the dashed line centered about $\lambda=1$. The backward traveling circuit wave is relatively uninfluenced by the presence of the electron beam, and is clearly identifiable in Fig. 9 as extending from 0 to $\pi$.

The series of drawings, Fig. 9(a) to 9(c), show the effect of operating voltage on the attenuation and phase constants of the system. Fig. 9(b) is for an operating voltage which places the slow space-charge wave approximately in synchronism with the forward traveling circuit wave in the middle of the pass band $\left(\theta_{0}+\theta_{\alpha}=3 \pi / 2\right)$. This operating voltage produces a maximum bandwidth. Growing and decaying wave solutions are obtained for frequencies below the low-frequency cutoff. For a : :omewhat higher voltage the situation depicted in Fig. 9(a), maximum rate of growth occurs at a frequency which is nearer the low cutoff frequency, and growing wave solutions are obtained at frequencies which are well below the low-freqency cutoff. Another interesting point is evident from this figure. The backward traveling creuit wave is actually nonpropagating at these low frequencies where growth is obtained. This effect makes it possible to operate near the low-frequency cutoff without oscillation in spite of the poor match which appears to exist at these frequencies. ${ }^{9} \mathrm{By}$ increasing the operating voltage still further, it is possible to make the backward attenuation exceed the forward growth. A traveling-wave tube amplifier employing this mode of operation should be stable for any input and output impedances. Results of this investigation seem to indicate that this mode of operation would be useful only for relatively narrow bandwidths, perhaps a few per cent. Reutz and Heffner are investigating this mode of operation in detail. ${ }^{10}$

Solution of the fourth-degree polynomial in $\mu$ gives only the attenuation constants and the phase constants of the four characteristic waves of the system. While a study of the attenuation constants and phase constants yields considerable insight into the characteristics of travelingwave tubes employing periodic circuits, in order to calculate the gain of such an amplifier it is necessary to determine the extent to which each of the characteristic waves is present. The four boundary conditions expressed by (14), (15), (44), and (45) lead to four linear algebraic equations in the constants $I_{01}, I_{02}, I_{03}$, and $I_{04}$. When these constants are known, the resonator current and voltage and the electron beam current may be calculated from (48)-(50). The power gain of the amplifier is defined as the ratio of the power dissipated in the load $R_{N+1}$ to the power available from the input generator,

power gain $=\frac{\left|I_{N=1}\right|^{2} R_{N+1}}{\left|V_{0}\right|^{2} / 4 R_{0}}=4\left|\frac{I_{N+1}}{V_{0}}\right|^{2} R_{0} R_{N+1}$.

In the figures the gain is expressed in decibels.

Fig. 10 shows gain vs frequency of a traveling-wave tube which employs a seven-resonator slow-wave circuit which has a 10 per cent bandwidth in the absence of the electron beam. The widest amplification bandwidth is obtained when the operating voltage is adjusted so that $\theta_{0}$ is approximately equal to $1.36 \pi$. Other parameters are approximately those which can be obtained with the slow-wave circuit of Fig. 1(a) and an electron beam of perveance $1.3 \times 10^{-6}$. The amplifier bandwidth is about 7 per cent at points where the gain is down 3 decibels. Operation at large signal levels tends to increase the bandwidth. At lower operating voltages $\left(\theta_{0}>1.36 \pi\right)$ maximum gain occurs near the upper cutoff frequency and gain near the lower cutoff frequency is reduced. This is explained simply by the dispersive character of the slow-wave circuit: near the high-frequency cutoff the wave travels at approximately the electron velocity, but near the low-frequency cutoff the wave velocity is much higher than the electron velocity. At operating voltages still lower than those represented in Fig. 10 the gain maxima at the upper cutoff frequency becomes very large and

${ }^{9}$ The electron beam actually alters the input and output impedances near the cutoffs significantly.

10 J. A. Ruetz and H. Heffner, "Consolidated Quarterly Status Report No. 1 on Electron Tube Research," Stanford University, Stanford, Calif.; March 31, 1957. 


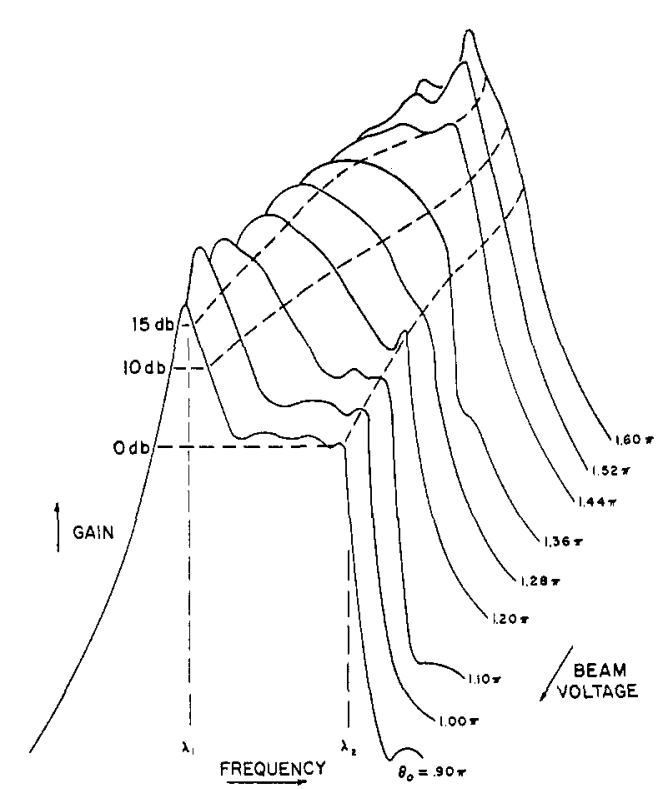

Fig. 10-Gain vs frequency of a seven-resonator traveling-wave tube with beam voltage as a parameter. $k=-0.05, q=0$, $\kappa=0.0033, \theta_{q}=0.13 \pi$.

the tube finally breaks into oscillation. For voltages which make $\theta_{0}$ fall between $2 \pi$ and $3 \pi$, the tube operates as a backward-wave oscillator. Its characteristics in this region are discussed later. When the operating voltage is greator than that which gives maximum bandwidth, the gain maximum is near the low-frequency cutoff and the gain near the high-frequency cutoff is reduced. This again is explained simply in terms of the dispersion of the slow-wave circuit. For the highest voltage shown in Fig. 10, maximum gain occurs at the low-frequency cutoff and is roughly symmetrical about that frequency. No gain is obtained in the upper half of the pass band, although the input signal is transmitted directly to the output in this frequency range. For the particular parameters chosen for this calculation, backward-wave oscillations do not occur when the operating voltage is increased to make $\theta_{0}$ less than $\pi$. A higher interaction impedance, higher beam current, or longer tube would be required to obtain backward-wave oscillations on this spatial harmonic.

Fig. 11 shows gain vs frequency of a nine-resonator circuit which has a 20 per cent bandwidth in the absence of the electron beam. Again the parameters correspond to those which can be obtained with the slow-wave circuit of Fig. 1(a), using an electron gun whose perveance is $1.3 \times 10^{-6}$. The three curves are for different operating voltages. Again, gain outside the pass band is clearly indicated. The center curve is for an operating voltage slightly higher than that which produces maximum bandwidth. The curve on the left is for a still higher operating voltage, while the curve on the right is for a lower operating voltage. The dashed curves show the gain calculated according to Birdsall and Brewer' ${ }^{11}$ large $C$ extension of

${ }^{11}$ C. K. Birdsall and G. R. Brewer, "Traveling wave tube characteristics for finite values of $C$," IRE Trass. ON Electron Devices, vol. ED-1, pp. 1-11; August, 1954.

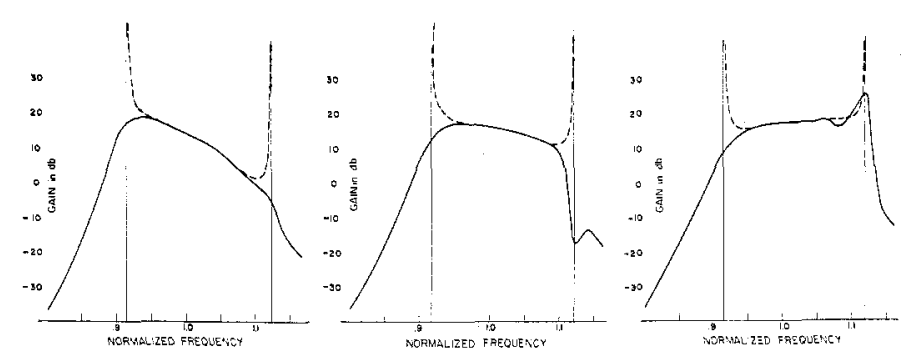

Fig. 11-Gain vs frequency of a nine-resonator traveling-wave tube with beam voltage as a parameter (solid curves). $k=-0.10$, $q=0, \kappa=0.0033, \theta_{0}=0.13 \pi, \theta_{0}=1.28 \pi$ (left), $1.36 \pi$ (center), $1,44 \pi$ (right). Dashed curves show gain predicted by Pierce theory.

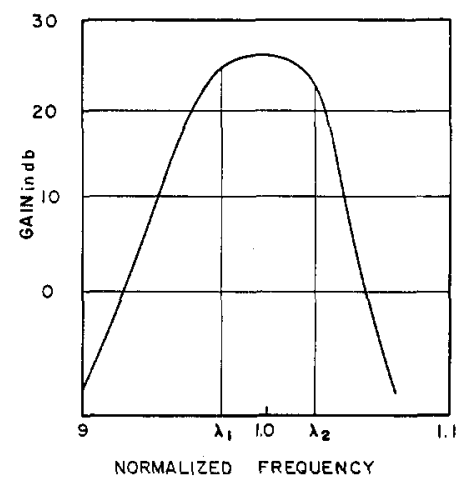

Fig. 12-Gain vs frequency of a five-resonator traveling-wave tube $\vec{k}=-0.025, q=0.01, \kappa=0.0118,0_{q}=0.18 \pi, \theta_{0}=1.32 \pi$.

Pierce's theory. The solid curves were obtained using the method outlined in this paper. It can be seen that the two methods give the same result near the middle of the pass band, but differ markedly near the two cutoff frequencies. As pointed out in the introduction, the infinite gain at the two cutoff frequencies in the Pierce theory arises from the infinite interaction impedance at those two points. However, since this theory does not include the possibility of simultaneous interaction with two independent spatial harmonics which can exist at either cutoff frequency, it is not surprising that the results obtained are incorrect. In the right-hand curve of Fig. 11, a small peak in the gain can be observed at the high frequency cutoff. As the operating voltage is reduced still further, this peak becomes very large and the tube finally breaks into oscillation. For still lower voltages, the tube operates as a backward-wave oscillator. This same effect also was pointed out in connection with Fig. 10.

Fig. 12 shows the frequency response of a travelingwave tube amplifier employing a five-resonator slow-wave circuit whose bandwidth is 5 per cent in the absence of the electron beam. The 3-db bandwidth of the amplifier is 5.5 per cent and a net gain is obtained over a 14 per cent bandwidth. It is important to remember that the calculated gain includes the effect of reflections from both the input and output transitions.

\section{Efficiency}

One important question about operation near the cutoff frequencies comes to mind immediately: What is the 

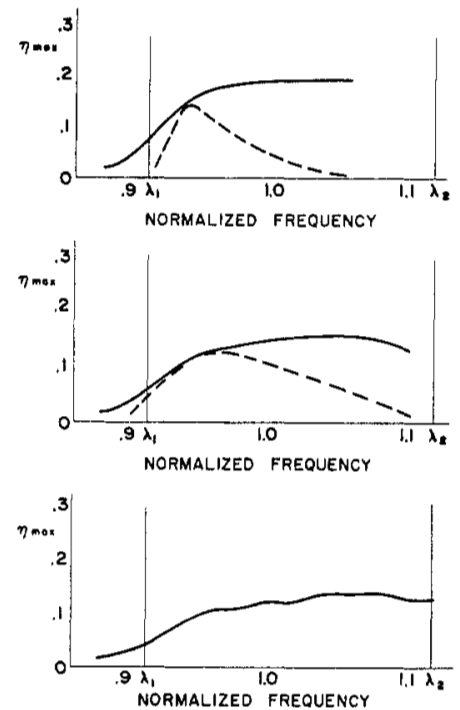

Fig. 13-Maximum efficiency of a nine-resonator travelijg-wave tube (solid curves) as estimated from the small-signal theory. $k=-0.10, q=0, \kappa=0.0033, \theta_{0}=0.13 \pi, \theta_{0}=1.28 \pi$ upper $1.36 \pi$ (center), $1.44 \pi$ (lower). Dashed curve shows varition in power output with constant input as calculated from the smallsignal theory.

electronic efficiency in this mode of operation? Since the interaction impedance of both modes near the cutoff frequencies become very large, one might hopefully expect that the electronic efficiency would be larger than obtained at midband. The electronic efficiency can be estimated from the small-signal theory of this model by calculating the power output when the alternating component of the beam current at the last resonator is equal to the $d:$ beam current. This method has been used previously but some caution must be exereised in interpreting the results. ${ }^{12}$ The results of this calculation are shown in Fig. 13. The three curves are for three different operating voltages. The dashed curves show the small signal gain under the same conditions for purposes of comparison. This figure indicates that the efficiency at the low-frequency cutoff may be somewhat less than the midband efficiency, while the efficiency at the upper cutoff frequency may be as large as the midband efficiency. Experimental measurements, on the other hand, seem to indicate that the efficiency at the low-frequency cutoff is practically as high as that which may be obtained at midband. It also may be seen from Fig. 11 that the maximum power output varies less with frequency than does the small sign il gain. In pulse applications this effect can be used to increase the amplification bandwidth somewhat.

\section{Backward-Wave Oscillation}

It is also of interest to study the characteristics of this periodic circuit traveling-wave tube as an oscillator operating on the backward spatial harmonics between 0 and $\pi$ phase shift, and between $2 \pi$ and $3 \pi$ phase shift. It is possible to use the method just described to find the

${ }^{12}$ R. Grow and D. A. Watkins, "Backward wave osci ator efficiency," Proc. IRE, vol. 43, pp. 848-856; July, 1955.
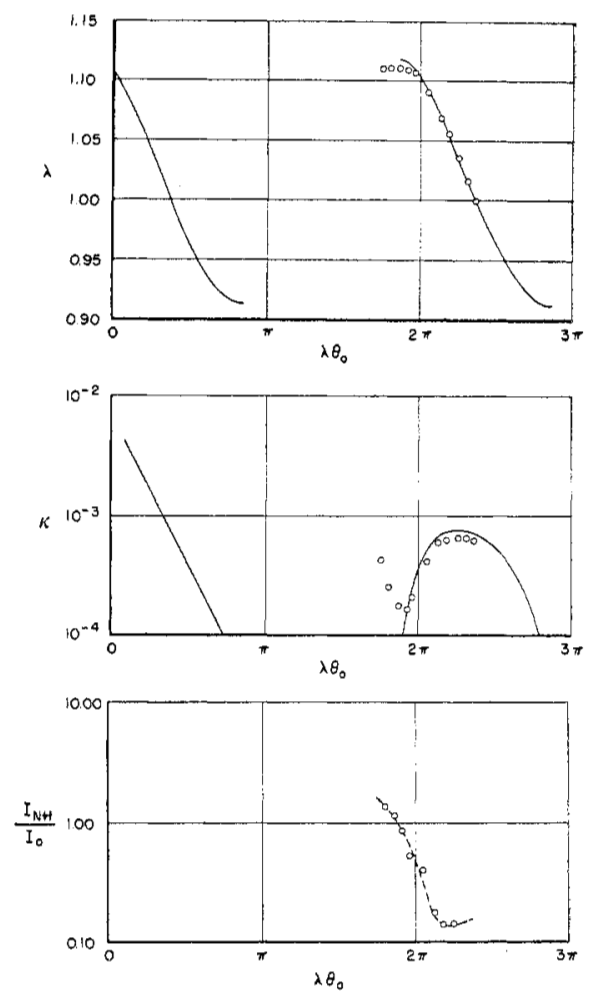

Fig. 14--Start oscillation conditions (circles) for a nine-resonator traveling-wave tube vs the beam voltage parameter $\lambda \theta_{0}$ : normalized oscillation frequency (upper curve), normalized starting current (center curve), and ratio of currents, input and output circuits (lower curve). $\theta_{q}=0.13 \pi, q=0, k=-0.10$. Solid lines are obtained from the theory of Johnson.

start oscillation conditions of a backward-wave oscillator. In the calculation to be described $k, q$, and $\theta_{q}$ are assumed to be constant and the variation of $Z_{0}$ and $Z_{N+1}$ with $\lambda$ is specified. For each value of $\theta_{0}$ there exists a value of $\lambda$ and $\kappa$ for which a finite voltage $V_{0}$ produces infinite input and output currents, $I_{0}$ and $I_{N+1}$. To put it another way, under these conditions finite input and output currents, $I_{0}$ and $I_{N+1}$ are obtained with zero input voltage. This is the condition at the start of oscillation. For larger values of $k$ the device oscillates spontaneously, while for smaller value of $\kappa$ there is no signal output. While it may be possible to choose the input circuit impedance $Z_{0}$ so that no signal is present in the impedance $Z_{N+1}$, in general a voltage is developed across both impedances. It will be interesting to examine the ratio of the currents $I_{N+1}$ to $I_{0}$, and see how it varies with frequency.

Fig. 14 shows how the frequency of oscillation $\lambda$ and the coupling constant $\kappa$ for the start of oscillation depend upon the operating voltage parameter $\theta_{0}$. The same nineresonator slow-wave circuit used in obtaining the data shown in Fig. 10 has been used although the terminating impedances have been changed slightly. For comparison, the start oscillation conditions have been computed from Johnson's paper ${ }^{13}$ and are shown by the solid lines. In Johnson's notation the space-charge parameter $H$ is 3.7 .

${ }^{13}$ H. R. Johnson, "Backward wave oscillators," Proc. IRE, vol. 43, pp. 684-697; June, 1955 . 
As may be expected, the tuning curves are in excellent agreement except for minor deviations near the cutoff frequencies. The coupling constants are only in moderate agreement in the central part of the pass band and no explanation is offered for the discrepancy. The usual theory of backward-wave oscillation predicts that the start oscillation current should approach zero as the cutoff frequencies are approached, because of the increasing circuit impedance. The analysis presented here on the other hand, predicts that the starting current decreases, reaches a minimum, and then increases again as the cutoff frequency is approached. This effect may again be attributed to the simultaneous interaction of the electron beam with two independent spatial harmonics. It is interesting to note that even when the operating voltage is such as to make the interaction primarily with the forward spatial harmonic, oscillation is still possible. Inspection of the ratio $I_{N+1} / I_{0}$ plotted in Fig. 14 shows that indeed, most of the output of the tube appears across the impedance $Z_{0}$. There is a continuous transition from backward-wave oscillation to forward-wave oscillation as the voltage is varied. With the proper impedances in the input and output circuits the forward wave start oscillation current can be made very high so that stable operation is achieved with the desired operating current. The effect of the input and output circuit impedances upon the backward-wave start oscillation current has not been investigated, although it is believed to be relatively minor.

\section{Transition to the Klystron Amplifier}

If the resonator coupling coefficient $k$ is reduced to obtain increased interaction impedance, with the consequent reduction in bandwidth, the traveling-wave tube pictured here begins to resemble a klystron. In the klystron the coupling between resonators is negligible $(k=0)$ but the resonator loss is not negligible ( $q$ cannot be taken to be zero). Whether the tube is a traveling-wave tube or a klystron depends then upon the ratio of the power flowing along the slow-wave circuit to the power dissipated in a resonator,

$$
\frac{P_{\text {transmitted }}}{P_{\text {dissipated }}}=\frac{\left.2 \nmid k\right|^{14}}{q}
$$

When this quantity is much larger than unity the device is clearly a traveling-wave tube, and when it is much less than unity the device is clearly a klystron. When the quantity is comparable to unity the device is hybrid.

When $k=0$, the fourth-degree polynomial in $\mu$ reduces to a second-degree polynomial whose solutions are

$$
\mu=e^{-i \lambda \theta_{0}}\left[\cos \theta_{q}+\xi \pm \sqrt{\left(\cos \theta_{q}+\xi\right)^{2}}-1\right]
$$

where

$$
\xi=\frac{\omega_{0}}{2 \omega_{p}} \kappa \sin \theta_{q} \frac{\lambda^{2}}{1-\lambda(\lambda-j q)}
$$

${ }^{14}$ This result is for midband $(\lambda=1)$ and is obtained with the aid of (31). It neglects the effect of the electron beam.
In the absence of interaction with the circuit $(\kappa=0)$ these two solutions represent the ordinary slow and fast space-charge waves. With interaction, one solution is an increasing wave and one solution is a decreasing wave. Maximum amplification occurs when $\theta_{a} \approx \pi / 2$. The absence of two waves simplifies calculations considerably, but it is questionable whether the method described has any advantages over conventional methods of analyzing multicavity klystrons. ${ }^{15}$

\section{Conclusion}

A lumped equivalent circuit of traveling-wave tubes which employ a certain class periodic slow-wave circuits has been presented and analyzed in some detail. This approach is useful in deriving and understanding some of the basic properties of periodic slow-wave circuits. Problems of the slow-wave circuit in the absence of the electron beam such as matching the slow-wave circuit to a uniform transmission line and the variation of circuit loss and interaction impedance with frequency may be usefully studied using the equivalent circuit. Of even more interest are the characteristics of a traveling-wave amplifier or oscillator employing such periodic slow-wave circuits. Frequency dependence of the gain has been studied, particularly near the cutoff frequencies, and several important effects are observed: finite and wellbehaved gain characteristics at a cutoff; useful amplification in a stopband, and appreciable modification of the matching impedance by the electron beam. Finite start oscillation current near cutoffs is also found and there is a continuous transition from backward-wave oscillation to forward-wave oscillation as the operating voltage is varied.

It is generally possible to obtain amplification without oscillation over an appreciable fraction of the pass band (sometimes including frequencies outside the pass band) at an operating voltage which makes the electron velocity approximately equal to the midband circuit wave velocity even though oscillation may occur at a cutoff for a reduced operating voltage. Thus in pulsed operation, oscillation may take place on the leading and trailing edges of the pulse unless the beam current is made zero or small during the rise and fall of the operating voltage.

\section{ACKNOWLEDGMENT}

It is a pleasure to acknowledge the interest and suggestions of members of the Electron Tube Laboratory of the Hughes Research and Development ILaboratories, particularly L. M. Field and S. Sensiper. The numerical results were obtained by E. A. Mazzari and J. Pochares, of the Hughes Systems Analysis Laboratory, using an IBM 650 digital computer. The author is deeply grateful for their considerable contribution to this study.

${ }_{15}$ When $k=0$ the coupling between the input circuit and first resonator and between the output circuit and last resonator is also zero. Thus, the equations for the first and last resonators must be rewritten or $k$ must be taken as small, but not zero, and the imped. ances $Z_{0}$ and $Z_{N+1}$ scaled appropriately. 